\title{
Lipschitz estimates for multilinear commutators of singular integral operators on spaces of homogeneous type
}

\author{
Chen Yue and Liu Lanzhe
}




\title{
LIPSCHITZ ESTIMATES FOR MULTILINEAR COMMUTATORS OF SINGULAR INTEGRAL OPERATORS ON SPACES OF HOMOGENEOUS TYPE
}

\author{
CHEN YUE AND LIU LANZHE
}

Received January 1, 2010

\begin{abstract}
In this paper we discuss the multilinear commutators of singular integral operators on spaces of homogeneous type. By using Hölder's inequalities and the $\left(L^{p}, L^{q}\right)$ type boundedness of fractional integral operators on spaces of homogeneous type, we obtain that this kind of multilinear commutators is bounded from $L^{p}$ to $L^{q}$, from $L^{p}$ to Triebel-Lizorkin spaces and from $H_{\vec{b}}^{p}$ to $L^{p}$. We also establish their $\left(H \dot{K}_{q_{1}, \vec{b}}^{\alpha, p}, \dot{K}_{q_{2}}^{\alpha, p}\right)$ type boundedness on spaces of homogeneous type.
\end{abstract}

2000 Mathematics Subject Classification: 42B20, 42B25

Keywords: singular integral operator, multilinear commutator, Lipschitz space, Hardy space, Herz-Hardy space, Triebel-Lizorkin space, space of homogeneous type

\section{INTRODUCTION}

As a development in the theory of singular integral operators, their commutators have been extensively studied in recent years. The results are plentiful and substantial. For instance, let $b \in B M O\left(R^{n}\right)$ and let $T$ be a Calderón-Zygmund operator. A classical result of Coifman, Rochberb and Weiss(see [9] states that the commutator $[b, T]$ is bounded on $L^{p}\left(R^{n}\right)(1<p<\infty)$. Chanillo (see [5]) proves a similiar result when $T$ is replaced by fractional operators (see [7,16]). The main purpose of this paper is to consider the continuity of the multilinear commutators associated with singular integral operators and Lipschitz functions in certain Hardy and Herz-Hardy spaces defined on spaces of homogeneous type. We also obtain the boundedness of the commutator $[b, T]$ generated by a singular integral operator $T$ and a Lipschitz function $b$ on Hardy and Herz type Hardy spaces. Not only that, we prove that this commutator is bounded from Hardy spaces to weak Lebesgue spaces and from Herz type Hardy spaces to weak Herz spaces.

First of all, let us introduce some definitions (see [1-6, 8, 14, 15]).

\footnotetext{
A Project Supported by Scientific Research Fund of Hunan Provincial Education Department $09 \mathrm{C} 057$.
} 
Give a set $X$, a function $d: X \times X \rightarrow R^{+}$is called a quasi-distance on $X$ if the following conditions are satisfied:

(i) for every $x$ and $y$ in $X, d(x, y) \geq 0$ and $d(x, y)=0$ if and only if $x=y$,

(ii) for every $x$ and $y$ in $X, d(x, y)=d(y, x)$,

(iii) there exists a constant $k \geq 1$ such that

$$
d(x, y) \leq k(d(x, z)+d(z, y))
$$

for every $x, y$ and $z$ in $X$.

Let $\mu$ be a positive measure on the $\sigma$-algebra of subsets of $X$ which contains the $r$-balls $B(x, r)=\{y: d(x, y)<r\}$. We assume that $\mu$ satisfies a doubling condition, that is, there exists a constant $A_{1}>1$ such that

$$
0<\mu(B(x, 2 r)) \leq A_{1} \mu(B(x, r))<\infty
$$

holds for all $x \in X$ and $r>0$.

A structure $(X, d, \mu)$, with $d$ and $\mu$ as above, is called a space of homogeneous type(Coifman and Weiss type). The constants $k$ and $A_{1}$ in (1.1) and (1.2) will be called the constants of the space.

From (1.2), we can say that there exists a constant $n_{0}>1$ such that $A_{1} \leq 2^{n_{0}}$, in other words, there exists a constant $n_{0}>1$ such that $\mu(B(x, 2 r)) \leq 2^{n_{0}} \mu(B(x, r))$.

We say that $(X, d, \mu)$ satisfies a reverse doubling condition, that is, there exists a constant $A_{2}>1$ such that

$$
0<A_{2} \mu(B(x, r)) \leq \mu(B(x, 2 r))<\infty
$$

holds for all $x \in X$ and $r>0$. It can be proved that, under some general additional geometric assumptions on the space $(X, d),(1.3)$ is actually a consequence of the doubling condition on $\mu$ (see [16]).

Definition 1 (see [12]). Let $d>0$ and $0<\theta \leq 1$. A space of homogeneous type $(X, \rho, \mu)_{d, \theta}$ is a set $\mathrm{X}$ together with a quasi-metric $\rho$ and a nonnegative Borel regular measure $\mu$ on X with supp $\mu=X$ and there exists a constant $C_{0}>1$ such that for all $0<r<\operatorname{diam} X$ and for all $x, x^{\prime}, y \in X$,

$$
\mu(B(x, r)) \sim r^{d}
$$

and

$$
\left|\rho(x, y)-\rho\left(x^{\prime}, y\right)\right| \leq C_{0} \rho\left(x, x^{\prime}\right)^{\theta}\left[\rho(x, y)+\rho\left(x^{\prime}, y\right)\right]^{1-\theta} .
$$

In this paper, we will consider this particular class of spaces of homogeneous type which satisfies the definitions of Coifman and Weiss and we will use $(X, \rho, \mu)_{d, \theta}$, too. Moreover, in [13], Macias and Segovia have proved that in the sense of equivalent topology, a space of homogeneous type in the sense of Coifman and Weiss is a space of homogeneous type in the sense of Definition 1 with $d=1$ and $\theta \in(0,1)$. 
Definition 2. For $\beta>0$, the Lipschitz space $\operatorname{Lip}_{\beta}(X)$ is the space of functions such that

$$
\|f\|_{\text {Lip }}=\sup _{\substack{x, y \in X \\ x \neq y}} \frac{|f(x)-f(y)|}{\rho(x, y)^{\beta}}<\infty .
$$

where $\rho$ is the quasi-distance.

Given a positive integer $m$ and $1 \leq i \leq m$, we denote by $C_{i}^{m}$ the family of all finite subsets $\sigma=\{\sigma(1), \ldots, \sigma(i)\}$ of $\{1, \ldots, m\}$ with $i$ different elements. For $\sigma \in C_{i}^{m}$, set $\sigma^{c}=\{1, \ldots, m\} \backslash \sigma$. Let $\vec{b}=\left(b_{1}, \ldots, b_{m}\right)$ and $\sigma=\{\sigma(1), \ldots, \sigma(i)\} \in C_{i}^{m}$, set $\vec{b}_{\sigma}=\left(b_{\sigma(1)}, \ldots, b_{\sigma(i)}\right)$ and $b_{\sigma}=b_{\sigma(1)} \ldots b_{\sigma(i)}$. With this notation, if $\beta_{\sigma(1)}+\cdots+$ $\beta_{\sigma(i)}=\beta_{\sigma}$, we write

$$
\left\|\vec{b}_{\sigma}\right\|_{L i p_{\beta \sigma}}=\left\|b_{\sigma(1)}\right\|_{L i p_{\beta_{\sigma(1)}}} \ldots|| b_{\sigma(i)} \|_{L i p_{\beta_{\sigma(i)}}} .
$$

For the product of all the functions, we simply write

$$
\left\|\vec{b}_{\sigma}\right\|_{L i p_{\beta}}=\prod_{i=1}^{m}\left\|b_{i}\right\|_{L i p_{\beta_{i}}},
$$

where $\sum_{i=1}^{m} \beta_{i}=\beta$.

Definition 3. Let $T$ be the singular integral operator

$$
T(f)(x)=\int_{X} K(x, y) f(y) d \mu(y),
$$

where $K$ is a locally integrable function on $X \times X \backslash\{(x, y) \mid x=y\}$ and satisfies the following properties:

(1) $|K(x, y)| \leq \frac{C}{\mu(B(x, d(x, y)))}$,

(2) there exists a $p_{0}, 1<p_{0} \leq \infty$, such that $T$ is bounded on $L^{p_{0}}(X)$,

(3) $\left|K(x, y)-K\left(x, y^{\prime}\right)\right|+\left|K(y, x)-K\left(y^{\prime}, x\right)\right| \leq C \frac{d\left(y, y^{\prime}\right)^{\delta}}{\mu(B(y, d(x, y))) d(x, y)^{\delta}}$, when $d(x, y) \geq 2 d\left(y, y^{\prime}\right)$, with some $\delta \in(0,1]$.

Similary to the definitions of other commutators, we introduce the definition of the commutator generated by a singular integral operator of Definition 3 and by a Lipschitz function as follows.

Definition 4. Let $\vec{b}=\left(b_{1}, \ldots, b_{m}\right)$ be a finite family of locally integrable functions with $b_{i} \in \wedge_{\beta}(X), 1 \leq i \leq m$ and lat $T$ be the singular integral operator. then we define the multilinear commutator as follows:

$$
T_{\vec{b}}(f)(x)=\int_{X} \prod_{i=1}^{m}\left(b_{i}(x)-b_{i}(y)\right) K(x, y) f(y) d \mu(y) .
$$


Note that when $b_{1}=\cdots=b_{m}, T_{\vec{b}}$ is just the $m^{\text {th }}$ order commutator. It is well known that commutators are of great interest in harmonic analysis and have been widely studied by many authors (see $[1-4,6,8]$ ).

We prove the boundedness ofthe commutator $T_{\vec{b}}$ on Lebesgue spaces in the following section.

\section{BOUNDEDNESS FROM $L^{p}(X)$ TO $L^{q}(X)$}

In this section, we obtain the following results.

Theorem 1. Let $b_{i} \in \operatorname{Lip}_{\beta_{i}}(X), 1 \leq i \leq m, \sum_{i=1}^{m} \beta_{i}=\beta, 0<\beta<1$ and $1 / q=$ $1 / p-\beta$. Then $T_{\vec{b}}$ is bounded from $L^{p}(X)$ to $L^{q}(X)$.

Theorem 2. Let $b_{i} \in \operatorname{Lip}_{\beta_{i}}(X), 1 \leq i \leq m, \sum_{i=1}^{m} \beta_{i}=\beta, 0<\beta<1$ and $1 / q=$ $1 / p-\beta$. Then $T_{\vec{b}}$ is bounded from $L^{1}(X)$ to weak $L^{1 /(1-\beta)}(X)$.

To prove the above results, we use the following lemmas.

Lemma 1 (see [10]). Let $0<\beta<1, f \in L^{p}(X)$ and $1 / q=1 / p-\beta$. Then $I_{\beta}$ is bounded from $L^{p}(X)$ to $L^{q}(X)$.

Lemma 2 (see [13]). Let $0<\beta<1, f \in L^{p}(X)$ and $1 / q=1 / p-\beta$. Then $I_{\beta}$ is bounded from $L^{1}(X)$ to weak $L^{1 /(1-\beta)}(X)$.

Here $I_{\beta}$ is the fractional integral operator.

Proof of Theorem 1 and 2. By Definition 4 and Definition 2, for any $x \in X$, we have

$$
\begin{aligned}
\left|T_{\vec{b}}(f)(x)\right| & \leq \int_{X} \prod_{i=1}^{m}\left|\left(b_{i}(x)-b_{i}(y)\right)\right||K(x, y) \| f(y)| d \mu(y) \\
& \leq \int_{X} \prod_{i=1}^{m}\left(\sup _{x \neq y} \frac{\left|b_{i}(x)-b_{i}(y)\right|}{d(x, y)^{\beta_{i}}} d(x, y)^{\beta_{i}}\right)|K(x, y) \| f(y)| d \mu(y) \\
& \leq\|\vec{b}\|_{\text {Lip }} \int_{X} d(x, y)^{\beta} \frac{|f(y)|}{\mu(B(x, d(x, y)))} d \mu(y) \\
& \leq\|\vec{b}\|_{\text {Lip }} \int_{X} \frac{|f(y)|}{\mu(B(x, d(x, y)))^{1-\beta}} d \mu(y) .
\end{aligned}
$$

So, by Lemma 1 and Lemma 2,

$$
\left\|T_{\vec{b}}(f)\right\|_{L^{q}} \leq C|| \vec{b}\left\|_{L i p_{\beta}}\right\| I_{\beta}(|f|)\left\|_{L^{q}} \leq C\right\| \vec{b}\left\|_{L i p_{\beta}}\right\| f \|_{L^{p}},
$$

and

$\mu\left(\left\{x \in X:\left|T_{\vec{b}}(f)(X)\right|>\lambda\right\}\right) \leq \mu\left(\left\{x \in X:\left|I_{\beta}(|f|)(x)\right|>\frac{\lambda}{C\|\vec{b}\|_{L i p_{\beta}}}\right\}\right)$ 


$$
\leq C\left(\|\vec{b}\|_{L i p_{\beta}} \frac{\|f\|_{L^{1}}}{\lambda}\right)^{1 / 1-\beta} \leq C\left(\frac{\|f\|_{L^{1}}}{\lambda}\right)^{1 / 1-\beta} .
$$

Thus we have finished the proof of the theorems.

\section{Boundedness From $L^{p}(X)$ TO TRIEbEL-Lizorkin SpaceS}

In this section, we are going to show that the multilinear commutator of a singular integral operator is bounded from $L^{p}(X)$ to Triebel-Lizorkin spaces.

Theorem 3. Let $b_{i} \in \operatorname{Lip}_{\beta_{i}}(X), 1 \leq i \leq m, \sum_{i=1}^{m} \beta_{i}=\beta, 0<\beta \leq \delta / n_{0}$ and $1 / q=1 / p-\beta$. Then $T_{\vec{b}}$ is bounded from $L^{p}(X)$ to $F_{p}^{\beta, \infty}(X)$.

To prove above theorems, we use some Lemmas.

Lemma 3 (see [13]). Let $0<\beta<1,1<p<\infty$, we have

$$
\begin{aligned}
\|f\|_{F_{p}^{\beta, \infty}} & =\left\|\sup _{B} \frac{1}{\mu(B)^{1+\beta}} \int_{B}\left|f(x)-f_{B}\right| d \mu(x)\right\|_{L^{p}} \\
& =\left\|\operatorname{supinf}_{B} \frac{1}{\mu(B)^{1+\beta}} \int_{B}|f(x)-c| d \mu(x)\right\|_{L^{p}}
\end{aligned}
$$

Lemma 4 (see [13]). Let $0<\beta<1,1 \leq p<\infty$, we have

$$
\begin{aligned}
\|f\|_{L i p_{\beta}} & =\sup _{B} \frac{1}{\mu(B)^{1+\beta}} \int_{B}\left|f(x)-f_{B}\right| d \mu(x) \\
& =\sup _{B} \mu(B)^{-\beta}\left[\frac{1}{\mu(B)} \int_{B}\left|f(x)-f_{B}\right|^{p} d \mu(x)\right]^{1 / p} .
\end{aligned}
$$

Lemma 5 (see [13]). Let balls $B_{1}, B_{2}$ with $B_{1} \subset B_{2}$.

Then $\left|f_{B_{1}}-f_{B_{2}}\right| \leq C\|f\|_{L_{i p_{\beta}}} \mu\left(B_{2}\right)^{\beta}$.

Proof of Theorem 3. Fix a ball $B=B\left(x_{0}, r\right)$ and $\tilde{x} \in B$. Set $\vec{b}_{B}=$ $\left(\left(b_{1}\right)_{B}, \ldots,\left(b_{m}\right)_{B}\right)$, where $\left(b_{i}\right)_{B}=\mu(B)^{-1} \int_{B} b_{i}(y) d \mu(y), 1 \leq i \leq m$. Write $f=$ $f_{1}+f_{2}$ with $f_{1}=f \chi_{2 B}$ and $f_{2}=f \chi_{(2 B)^{c}}$, then we have

$$
\begin{aligned}
& T_{\vec{b}}(f)(x)=\int_{X} \prod_{i=1}^{m}\left(b_{i}(x)-b_{i}(y)\right) K(x, y) f(y) d \mu(y) \\
& =\int_{X} \prod_{i=1}^{m}\left(\left(b_{i}(x)-\left(b_{i}\right)_{B}\right)+\left(\left(b_{i}\right)_{B}-b_{i}(y)\right)\right) K(x, y) f(y) d \mu(y) \\
& \leq C \sum_{i=0}^{m} \sum_{\sigma \in C_{i}^{m}}(-1)^{m-i}\left(\vec{b}(x)-\vec{b}_{B}\right)_{\sigma} \int_{X}\left(\vec{b}(y)-\vec{b}_{B}\right)_{\sigma^{c}} K(x, y) f(y) d \mu(y)
\end{aligned}
$$




$$
\begin{aligned}
& \leq C \prod_{i=1}^{m}\left(b_{i}(x)-\left(b_{i}\right)_{B}\right) T(f)(x) \\
& +\sum_{i=1}^{m-1} \sum_{\sigma \in C_{i}^{m}}(-1)^{m-i}\left(\vec{b}(x)-\vec{b}_{B}\right)_{\sigma} T\left(\left(\vec{b}-\vec{b}_{B}\right)_{\sigma^{c}} f\right)(x) \\
& +(-1)^{m} T\left(\prod_{i=1}^{m}\left(b_{i}-\left(b_{i}\right)_{B}\right) f\right)(x) .
\end{aligned}
$$

So, let $c=T\left(\prod_{i=1}^{m}\left(b_{i}-\left(b_{i}\right)_{B}\right) f_{2}\right)\left(x_{0}\right)$. It is easy to see now that

$$
\begin{aligned}
& \frac{1}{\mu(B)^{1+\beta}} \int_{B}\left|T_{\vec{b}}(f)(x)-c\right| d \mu(x) \\
& \leq \frac{1}{\mu(B)^{1+\beta}} \int_{B}\left|T_{\vec{b}}(f)(x)-T\left(\prod_{i=1}^{m}\left(b_{i}-\left(b_{i}\right)_{B}\right) f_{2}\right)\left(x_{0}\right)\right| d \mu(x) \\
& \leq \frac{C}{\mu(B)^{1+\beta}} \int_{B}\left|\prod_{i=1}^{m}\left(b_{i}(x)-\left(b_{i}\right)_{B}\right) T(f)(x)\right| d \mu(x) \\
& +\frac{C}{\mu(B)^{1+\beta}} \int_{B}\left|\sum_{i=1}^{m-1} \sum_{\sigma \in C_{i}^{m}}\left(\vec{b}(x)-\vec{b}_{B}\right)_{\sigma} T\left(\left(\vec{b}-\vec{b}_{B}\right)_{\sigma^{c}} f\right)(x)\right| d \mu(x) \\
& +\frac{C}{\mu(B)^{1+\beta}} \int_{B}\left|T\left(\prod_{i=1}^{m}\left(b_{i}-\left(b_{i}\right)_{B}\right) f_{1}\right)(x)\right| d \mu(x) \\
& +\frac{C}{\mu(B)^{1+\beta}} \int_{B}\left|T\left(\prod_{i=1}^{m}\left(b_{i}(x)-\left(b_{i}\right)_{B}\right) f_{2}\right)(x)-T\left(\prod_{i=1}^{m}\left(b_{i}-\left(b_{i}\right)_{B}\right) f_{2}\right)\left(x_{0}\right)\right| d \mu(x) \\
& =I+I I+I I I+I V .
\end{aligned}
$$

For the term $I$, by Lemma 4 and by Hölder's inequalities with exponent $1 / q_{1}+\cdots+$ $1 / q_{m}+1 / q=1, q>1, q_{i}>1(i=1, \ldots, m)$, we have

$$
\begin{aligned}
I & \leq \frac{C}{\mu(B)^{1+\beta}} \prod_{i=1}^{m}\left(\int_{B}\left|b_{i}(x)-\left(b_{i}\right)_{B}\right|^{q_{i}} d \mu(x)\right)^{1 / q_{i}}\left(\int_{B}|T(f)(x)|^{q} d \mu(x)\right)^{1 / q} \\
& \leq C\|\vec{b}\|_{L i p_{\beta}} M_{q}(|T(f)|)(\tilde{x})
\end{aligned}
$$

For the term $I I$, by Lemma 4, by Hölder's inequalities with exponent $1 / s+1 / s^{\prime}=1$, $1 / q+1 / q^{\prime}=1$ and by the $L^{p}(1<p<\infty)$ boundedness of $T$, we have

$$
I I \leq \frac{C}{\mu(B)^{1+\beta}} \sum_{i=1}^{m-1} \sum_{\sigma \in C_{i}^{m}} \int_{B}\left|\left(\vec{b}(x)-\vec{b}_{B}\right)_{\sigma} T\left(\left(\vec{b}-\vec{b}_{B}\right)_{\sigma^{c}} f\right)(x)\right| d \mu(x)
$$




$$
\begin{aligned}
& \leq \frac{C}{\mu(B)^{1+\beta}} \sum_{i=1}^{m-1} \sum_{\sigma \in C_{i}^{m}}\left(\int_{B}\left|\left(\vec{b}(x)-\vec{b}_{B}\right)_{\sigma}\right|^{q^{\prime}} d \mu(x)\right)^{1 / q^{\prime}} \\
& \times\left(\int_{B}\left|T\left(\left(\vec{b}-\vec{b}_{B}\right)_{\sigma^{c}} f\right)(x)\right|^{q} d \mu(x)\right)^{1 / q} \\
& \leq C \sum_{i=1}^{m-1} \sum_{\sigma \in C_{i}^{m}} \mu(B)^{-\beta+\beta_{\sigma(1)}+\cdots+\beta_{\sigma(i)}}\left\|\vec{b}_{\sigma}\right\|_{L i p_{\beta}} \\
& \times\left(\frac{1}{\mu(B)} \int_{B}\left|\left(\vec{b}(x)-\vec{b}_{B}\right)_{\sigma^{c}} f(x)\right|^{q} d \mu(x)\right)^{1 / q} \\
& \leq C \sum_{i=1}^{m-1} \sum_{\sigma \in C_{i}^{m}} \mu(B)^{-\beta+\beta_{\sigma(1)}+\cdots+\beta_{\sigma(i)}\left\|\vec{b}_{\sigma}\right\|_{L i p_{\beta}}} \\
& \times\left(\frac{1}{\mu(B)} \int_{B}\left|\left(\vec{b}(x)-\vec{b}_{B}\right)_{\sigma^{c}}\right|^{s^{\prime} q} d \mu(x)\right)^{1 / s^{\prime} q}\left(\frac{1}{\mu(B)} \int_{B}|f(x)|^{s q} d \mu(x)\right)^{1 / s q} \\
& \leq C\|\vec{b}\|_{L i p_{\beta}} M_{s q}(f)(\tilde{x}) .
\end{aligned}
$$

For the term $I I I$, by the $L^{p}(1<p<\infty)$ boundedness of $T$ and by Hölder's inequalities with exponent $1 / s_{1}+\cdots+1 / s_{m}+1 / s=1, s_{i}>1, i=1, \ldots, m, s>1$, we have

$$
\begin{aligned}
I I I & \leq \frac{1}{\mu(B)^{1+\beta}} \int_{B}\left|T\left(\prod_{i=1}^{m}\left(b_{i}-\left(b_{i}\right)_{B}\right) f_{1}\right)(x)\right| d \mu(x) \\
& \leq C \mu(B)^{-\beta}\left(\frac{1}{\mu(B)} \int_{X}\left|T\left(\prod_{i=1}^{m}\left(b_{i}-\left(b_{i}\right)_{B}\right) f_{1}\right)(x)\right|^{q} \chi_{B} d \mu(x)\right)^{1 / q} \\
& \leq C \mu(B)^{-\beta}\left(\frac{1}{\mu(B)} \int_{B}\left|\prod_{i=1}^{m}\left(b_{i}(x)-\left(b_{i}\right)_{B}\right) f(x)\right|^{q} d \mu(x)\right)^{1 / q} \\
& \leq C \prod_{i=1}^{m} \mu(B)^{-\beta_{i}}\left(\frac{1}{\mu(B)} \int_{B}\left|\left(b_{i}(x)-\left(b_{i}\right)_{B}\right)\right|^{s_{i} q} d \mu(x)\right)^{1 / s_{i} q} \\
& \times\left(\frac{1}{\mu(B)} \int_{B}|f(x)|^{s q} d \mu(x)\right)^{1 / s q} \\
& \leq C\|\vec{b}\|_{L i p_{\beta}} M_{s q}(f)(\tilde{x}) .
\end{aligned}
$$

For the term $I V$, by Hölder's inequalities with exponent $1 / q^{\prime}+1 / q=1$, we get

$$
\int_{X} \prod_{i=1}^{m}\left|\left(b_{i}(y)-\left(b_{i}\right)_{B}\right)\right|\left|K(x, y)-K\left(x_{0}, y\right)\right|\left|f_{2}(y)\right| d \mu(y)
$$




$$
\begin{aligned}
& \leq \int_{(2 B)^{c}} \prod_{i=1}^{m}\left|\left(b_{i}(y)-\left(b_{i}\right)_{B}\right)\right| \frac{d\left(x, x_{0}\right)^{\delta}}{d\left(x_{0}, y\right)^{\delta} \mu\left(B\left(x_{0}, d\left(x_{0}, y\right)\right)\right)}|f(y)| d \mu(y) \\
& \leq C \sum_{k=1}^{\infty} \frac{d\left(x, x_{0}\right)^{\delta}}{\left(2^{k} r\right)^{\delta} \mu\left(2^{k} B\right)} \int_{2^{k+1} B} \prod_{i=1}^{m}\left|\left(b_{i}(y)-\left(b_{i}\right)_{B}\right) \| f(y)\right| d \mu(y) \\
& \leq C \sum_{k=1}^{\infty} \frac{d\left(x, x_{0}\right)^{\delta}}{\left(2^{k} r\right)^{\delta} \mu\left(2^{k} B\right)} \sum_{i=0}^{m} \sum_{\sigma \in C_{i}^{m}}\left|\left(\vec{b}_{2^{k+1} B}-\vec{b}_{B}\right)_{\sigma^{c}}\right| \\
& \times \int_{2^{k+1} B}\left|\left(\vec{b}(y)-\vec{b}_{2^{k+1} B}\right)_{\sigma}\right||f(y)| d \mu(y) \\
& \leq C \sum_{k=1}^{\infty} \frac{d\left(x, x_{0}\right)^{\delta}}{\left(2^{k} r\right)^{\delta} \mu\left(2^{k} B\right)} \sum_{i=0}^{m} \sum_{\sigma \in C_{i}^{m}}\left|\left(\vec{b}_{2^{k+1} B}-\vec{b}_{B}\right)_{\sigma^{c}}\right| \\
& \times\left(\int_{2^{k+1} B}\left|\left(\vec{b}(y)-\vec{b}_{2^{k+1} B}\right)_{\sigma}\right|^{q^{\prime}} d \mu(y)\right)^{1 / q^{\prime}}\left(\int_{2^{k+1} B}|f(y)|^{q} d \mu(y)\right)^{1 / q} \\
& \leq C \sum_{k=1}^{\infty} \sum_{i=0}^{m} \sum_{\sigma \in C_{i}^{m}} \frac{d\left(x, x_{0}\right)^{\delta}}{\left(2^{k} r\right)^{\delta} \mu\left(2^{k} B\right)} \\
& \times\left\|\vec{b}_{\sigma^{c}}\right\|_{L i p_{\beta}} \mu\left(2^{k+1} B\right)^{\beta_{\sigma^{c}}} \mu\left(2^{k+1} B\right)^{\beta_{\sigma}}\left\|\vec{b}_{\sigma}\right\|_{\wedge_{\beta}} M_{q}(|f|)(\tilde{x}) \mu\left(2^{k+1} B\right) \\
& \leq C\|\vec{b}\|_{L i p_{\beta}} M_{q}(f)(\tilde{x}) \sum_{k=1}^{\infty} \frac{d\left(x, x_{0}\right)^{\delta}}{\left(2^{k} r\right)^{\delta} \mu\left(2^{k} B\right)} \mu\left(2^{k+1} B\right)^{1+\beta} \text {, }
\end{aligned}
$$

so,

$$
\begin{aligned}
I V & \leq C\|\vec{b}\|_{L i p_{\beta}} M_{q}(f)(\tilde{x}) \frac{1}{\mu(B)^{1+\beta}} \sum_{k=1}^{\infty} \mu\left(2^{k+1} B\right)^{1+\beta} \int_{B} \frac{d\left(x, x_{0}\right)^{\delta}}{\left(2^{k} r\right)^{\delta} \mu\left(2^{k} B\right)} d \mu(x) \\
& \leq C\|\vec{b}\|_{L i p_{\beta}} M_{q}(f)(\tilde{x}) \sum_{k=1}^{\infty} 2^{-k \delta} \mu(B)^{-\beta} \mu\left(2^{k} B\right)^{\beta} \\
& \leq C\|\vec{b}\|_{L i p_{\beta}} M_{q}(f)(\tilde{x}) \sum_{k=1}^{\infty} 2^{k n_{0}\left(\beta-\delta / n_{0}\right)} \\
& \leq C\|\vec{b}\|_{L i p_{\beta}} M_{q}(f)(\tilde{x}) .
\end{aligned}
$$

We put these estimates together, by using Lemma 3 and taking the supremum over all $B$ such that $\tilde{x} \in B$, we obtain

$$
\begin{aligned}
\|f\|_{F_{p}^{\beta, \infty}} & \leq C\|\vec{b}\|_{L i p_{\beta}}\left(\left\|M_{q}(T(f))\right\|_{L^{p}}+\left\|M_{q s}(f)\right\|_{L^{p}}+\left\|M_{q}(f)\right\|_{L^{p}}\right) \\
& \leq C\|\vec{b}\|_{L i p_{\beta}}\|f\|_{L^{p}}
\end{aligned}
$$


Thus we have finished the proof of the theorem.

\section{Boundedness on HaRdy SPACES}

In this section, we discuss the boundedness of the multiliner commutator $T_{\vec{b}}$ generated by the singular integral operator $T$ and the Lipschitz function $\vec{b}$ on Hardy spaces.

First, let us review the atomic decomposition of Hardy spaces, which is important in the study the boundedness of operators.

Definition 5. Let $b_{i}(i=1, \cdots, m)$ be locally integrable functions and $0<p \leq 1$. A bounded measurable function $a(x)$ on $X$ is called a $(p, \vec{b})$ atom, if

(1) supp $a \subset B=B\left(x_{0}, r\right)$,

(2) $\|a\|_{L^{\infty}} \leq \mu(B)^{-1 / p}$,

(3) $\int_{B} a(y) d \mu(y)=\int_{B} a(y) \prod_{l \in \sigma} b_{l}(y) d \mu(y)=0$ for any $\sigma \in C_{j}^{m}, 1 \leq j \leq m$.

A tempered distribution $f$ is said to belong to the atomic space $H_{\vec{b}}^{p}(X)$, if, in the Schwartz distribution sense, it can be written as $f(x)=\sum_{i} \lambda_{i} a_{i}(x)$, where $a_{i}$ are $(p, \vec{b})$ atoms, $\lambda_{i} \in C$ and $\sum_{i}\left|\lambda_{i}\right|^{p}<\infty$. Moreover, $\|f\|_{H_{\vec{b}}^{p}}=\inf \left(\sum_{i}\left|\lambda_{i}\right|^{p}\right)^{1 / p}$, where the infimum are taken over all of the above decompositions of $f$.

Theorem 4. Let $b_{i} \in \operatorname{Lip}_{\beta_{i}}(X), 1 \leq i \leq m, \sum_{i=1}^{m} \beta_{i}=\beta, 0<\beta \leq 1,0<p \leq 1$ and $1 / q=1 / p-\beta$. Then $T_{\vec{b}}$ is bounded from $H_{\vec{b}}^{p}(X)$ to $L^{q}(X)$.

Theorem 5. Let $b_{i} \in \operatorname{Lip}_{\beta_{i}}(X), 1 \leq i \leq m, \sum_{i=1}^{m} \beta_{i}=\beta, 0<\beta \leq \delta / n_{0}$ and $0<p \leq 1$. Then $T_{\vec{b}}$ is bounded from $H_{\vec{b}}^{1 /(1+\beta)}(X)$ to weak $L^{1}(X)$.

Proof of Theorem 4. By a standard argument, it suffices to show that there exists a constants $C>0$ such that $\left\|T_{\vec{b}}(a)\right\|_{L^{q}} \leq C$ for each $(p, \vec{b})$ atom $a$.

$$
\begin{gathered}
\left\|T_{\vec{b}}(a)\right\|_{L^{q}} \leq\left(\int_{2 B}\left|T_{\vec{b}}(a)(x)\right|^{q} d \mu(x)\right)^{1 / q}+\left(\int_{(2 B)^{c}}\left|T_{\vec{b}}(a)(x)\right|^{q} d \mu(x)\right)^{1 / q} \\
=I+I I
\end{gathered}
$$

For the term $I$, taking $q>1$, by Hölder's inequality and by Theorem 1, we get

$$
I \leq C\left\|T_{\vec{b}}(a)\right\|_{L^{q}} \leq C\|\vec{b}\|_{L i p_{\beta}}\|a\|_{L^{p}} \leq C\|\vec{b}\|_{L i p_{\beta}}\|a\|_{L^{\infty}} \cdot \mu(B)^{1 / p} \leq C .
$$

For the term $I I$, for $y \in B, x \in(2 B)^{c}$, by the vanishing of the moment of $a$ and by Hölder's inequalities, we get

$$
\left|T_{\vec{b}}(a)(x)\right|=\int_{B} \prod_{i=1}^{m}\left|\left(b_{i}(x)-b_{i}(y)\right)\right||K(x, y)||a(y)| d \mu(y)
$$




$$
\begin{aligned}
& \leq \int_{B} \prod_{i=1}^{m}\left|\left(b_{i}(x)-b_{i}\left(x_{0}\right)\right)+\left(b_{i}\left(x_{0}\right)-b_{i}(y)\right)\right| \\
& \left|K(x, y)-K\left(x, x_{0}\right)\right||a(y)| d \mu(y) \\
& \leq \prod_{i=1}^{m}\left|b_{i}(x)-b_{i}\left(x_{0}\right)\right| \int_{B}\left|K(x, y)-K\left(x, x_{0}\right)\right||a(y)| d \mu(y) \\
& +\sum_{i=1}^{m-1} \sum_{\sigma \in C_{i}^{m}}\left|\left(\vec{b}(x)-\vec{b}\left(x_{0}\right)\right)_{\sigma}\right| \\
& \int_{B}\left|\left(\vec{b}(y)-\vec{b}\left(x_{0}\right)\right)_{\sigma^{c}}\right|\left|K(x, y)-K\left(x, x_{0}\right)\right||a(y)| d \mu(y) \\
& +\int_{B} \prod_{i=1}^{m}\left|b_{i}(y)-b_{i}\left(x_{0}\right)\right|\left|K(x, y)-K\left(x, x_{0}\right)\right||a(y)| d \mu(y) \\
& \leq C \frac{r^{\delta}}{d\left(x, x_{0}\right)^{\delta} \mu\left(B\left(x_{0}, d\left(x, x_{0}\right)\right)\right)} \cdot\left\{\|\vec{b}\|_{L i p_{\beta}} \rho\left(x, x_{0}\right)^{\beta}\|a\|_{L^{\infty} \mu(B)}\right. \\
& +\sum_{i=1}^{m-1} \sum_{\sigma \in C_{i}^{m}}\left\|\vec{b}_{\sigma}\right\|_{L i p_{\beta}} \rho\left(x, x_{0}\right)^{\beta_{\sigma}} \int_{B}\left\|\vec{b}_{\sigma_{c}}\right\|_{L i p_{\beta}} \rho\left(y, x_{0}\right)^{\beta_{\sigma} c}|a(y)| d \mu(y) \\
& \left.+\int_{B}\|\vec{b}\|_{L i p_{\beta}} \rho\left(y, x_{0}\right)^{\beta}|a(y)| d \mu(y)\right\} \\
& \leq C \frac{r^{\delta}}{d\left(x, x_{0}\right)^{\delta} \mu\left(B\left(x_{0}, d\left(x, x_{0}\right)\right)\right)}\|\vec{b}\|_{L i p_{\beta}} \rho\left(x, x_{0}\right)^{\beta}\|a\|_{L^{\infty} \mu(B)} \\
& \leq C \frac{r^{\delta}}{d\left(x, x_{0}\right)^{\delta} \mu\left(B\left(x_{0}, d\left(x, x_{0}\right)\right)\right)}\|\vec{b}\|_{L i p_{\beta}} \rho\left(x, x_{0}\right)^{\beta} \mu(B)^{1 / p^{\prime}},
\end{aligned}
$$

so, we have

$$
\begin{aligned}
& I I^{q}=\int_{(2 B)^{c}}\left|T_{\vec{b}}(a)(x)\right|^{q} d \mu(x) \\
& \leq C \int_{(2 B)^{c}} \frac{r^{\delta q}}{d\left(x, x_{0}\right)^{\delta q} \mu\left(B\left(x_{0}, d\left(x, x_{0}\right)\right)\right)^{q}}\|\vec{b}\|_{L i p_{\beta}}^{q} \rho\left(x, x_{0}\right)^{\beta q} \mu(B)^{q / p^{\prime}} d \mu(x) \\
& \leq C\|\vec{b}\|_{L i p_{\beta}}^{q} \sum_{k=1}^{\infty} \int_{2^{k+1} B} \frac{r^{\delta q}}{\left(2^{k} r\right)^{\delta q} \mu\left(2^{k} B\right)^{q}} \mu\left(2^{k} B\right)^{\beta q} \mu(B)^{q / p^{\prime}} d \mu(x)
\end{aligned}
$$




$$
\begin{aligned}
& \leq C\|\vec{b}\|_{L i p_{\beta}}^{q} \sum_{k=1}^{\infty} 2^{-k \delta q} \mu(B)^{q / p^{\prime}} \mu\left(2^{k} B\right)^{q(-1+1 / p)} \\
& \leq C\|\vec{b}\|_{L i p_{\beta}}^{q} .
\end{aligned}
$$

Thus, we have finished the proof of the theorem.

Proof of Theorem 5. By the atomic decomposition, we know that there exist $(1 /(1+$ $\beta), \vec{b}$ )-atom $a_{j}$, supp $a_{j} \in B_{j}=B\left(x_{j}, r_{j}\right)$, so, we obtain that

$$
\begin{aligned}
\left\|T_{\vec{b}}(f)\right\|_{L^{1}} & \leq \int_{2 B_{j}} T_{\vec{b}}(f)(x) d \mu(x)+\int_{(2 B)^{c}} T_{\vec{b}}(f)(x) d \mu(x) \\
& =M_{1}+M_{2} .
\end{aligned}
$$

For $M_{1}$, by the Minkowski and Hölder inequalities, by the $\left(L^{p}, L^{p}\right)$-boundedness of $T$ and by Definition 1, we have

$$
\begin{aligned}
\left|M_{1}\right| & =C \sum_{j=-\infty}^{\infty}\left|\lambda_{j}\right| \int_{2 B_{j}}\left|T_{\vec{b}}\left(a_{j}\right)(x)\right| d \mu(x) \\
& \leq C \sum_{j=-\infty}^{\infty}\left|\lambda_{j}\right| \int_{2 B_{j}} \int_{B_{j}} \prod_{i=1}^{m}\left|b_{i}(x)-b_{i}(y)\|K(x, y)\| a_{j}(y)\right| d \mu(y) d \mu(x) \\
& \leq C \sum_{j=-\infty}^{\infty}\left|\lambda_{j}\right| \int_{2 B_{j}} \int_{B_{j}}\|\vec{b}\|_{\wedge_{\beta}} d(x, y)^{\beta}\left|K(x, y) \| a_{j}(y)\right| d \mu(y) d \mu(x) \\
& \leq C\|\vec{b}\|_{L i p_{\beta}} \sum_{j=-\infty}^{\infty}\left|\lambda_{j}\right| \int_{2 B_{j}} d\left(x, x_{0}\right)^{\beta}\left|T\left(a_{j}\right)(x)\right| d \mu(x) \\
& \leq C\|\vec{b}\|_{L i p_{\beta}} \sum_{j=-\infty}^{\infty}\left|\lambda_{j}\right| r_{j}{ }^{\beta}\left\|T\left(a_{j}\right)\right\|_{L^{p}} \mu\left(2 B_{j}\right)^{1 / p^{\prime}} \\
& \leq C\|\vec{b}\|_{L i p_{\beta}} \sum_{j=-\infty}^{\infty}\left|\lambda_{j}\right| r_{j}{ }^{\beta}\left\|a_{j}\right\|_{L^{p}} \mu\left(2 B_{j}\right)^{1 / p^{\prime}} \\
& \leq C\|\vec{b}\|_{L i p_{\beta}} \sum_{j=-\infty}^{\infty}\left|\lambda_{j}\right|
\end{aligned}
$$

For $M_{2}$, similary to case $I I$ in Theorem 4 and by Definition 5, we have

$$
T_{\vec{b}}(f)(x) \leq C \sum_{j=-\infty}^{\infty} \lambda_{j} \prod_{i=1}^{m}\left|b_{i}(x)-b_{i}\left(x_{0}\right)\right| \int_{B_{j}}\left|K(x, y)-K\left(x, x_{0}\right)\right|\left|a_{j}(y)\right| d \mu(y)
$$




$$
\begin{aligned}
& +\sum_{j=-\infty}^{\infty}\left|\lambda_{j}\right| \sum_{i=1}^{m-1} \sum_{\sigma \in C_{i}^{m}}\left|\left(\vec{b}(x)-\vec{b}\left(x_{0}\right)\right)_{\sigma}\right| \\
& \int_{B_{j}}\left|\left(\vec{b}(y)-\vec{b}\left(x_{0}\right)\right)_{\sigma^{c}}\right|\left|K(x, y)-K\left(x, x_{0}\right)\right|\left|a_{j}(y)\right| d \mu(y) \\
& +\sum_{j=-\infty}^{\infty}\left|\lambda_{j}\right| \prod_{i=1}^{m} \int_{B_{j}}\left|b_{i}(y)-b_{i}\left(x_{0}\right)\right|\left|K(x, y)-K\left(x, x_{0}\right)\right|\left|a_{j}(y)\right| d \mu(y) \\
& \quad \leq C \sum_{j=-\infty}^{\infty}\left|\lambda_{j}\right||\vec{b}|_{L i p_{\beta}} \frac{r_{j}{ }^{\delta}}{d\left(x, x_{0}\right)^{\delta} \mu\left(B\left(x_{0}, d\left(x, x_{0}\right)\right)\right)} \rho\left(x, x_{0}\right)^{\beta}\left\|a_{j}\right\|_{L^{\infty}} \mu\left(B_{j}\right) \\
& \quad \leq C \sum_{j=-\infty}^{\infty}\left|\lambda_{j}\right| \mid \vec{b} \|_{L i p_{\beta}} \frac{r_{j}{ }^{\delta}}{d\left(x, x_{0}\right)^{\delta} \mu\left(B\left(x_{0}, d\left(x, x_{0}\right)\right)\right)} \rho\left(x, x_{0}\right)^{\beta} \mu\left(B_{j}\right)^{-\beta}
\end{aligned}
$$

so,

$$
\begin{aligned}
M_{2} \leq & C \sum_{j=-\infty}^{\infty}\left|\lambda_{j}\right| \mu\left(B_{j}\right)^{-\beta} \\
& \sum_{k=1}^{\infty} \int_{2^{k+1} B_{j} \backslash 2^{k} B_{j}} \frac{r_{j}^{\delta}}{d\left(x, x_{0}\right)^{\delta} \mu\left(B\left(x_{0}, d\left(x, x_{0}\right)\right)\right)} \rho\left(x, x_{0}\right)^{\beta} d \mu(x) \\
& \leq C \sum_{j=-\infty}^{\infty}\left|\lambda_{j}\right| \mu\left(B_{j}\right)^{-\beta} \sum_{k=1}^{\infty} \frac{r_{j}^{\delta}}{\left(^{k} r_{j}\right)^{\delta} \mu\left(2^{k} B_{j}\right)} \mu\left(2^{k+1} B_{j}\right)^{1+\beta} \\
& \leq C \sum_{j=-\infty}^{\infty}\left|\lambda_{j}\right| \mu\left(B_{j}\right)^{-\beta} \sum_{k=1}^{\infty} 2^{-k \delta} \mu\left(2^{k} B_{j}\right)^{\beta} \\
& \leq C \sum_{j=-\infty}^{\infty}\left|\lambda_{j}\right| \sum_{k=1}^{\infty} 2^{k\left(-\delta+n_{0} \beta\right)} \\
& \leq C \sum_{j=-\infty}^{\infty}\left|\lambda_{j}\right| .
\end{aligned}
$$

Therefore, by the Jensen inequality, we have

$$
\begin{aligned}
\mu\left(x \in X:\left|T_{\vec{b}}(f)\right|>\lambda\right) & \leq C \lambda^{-1}\left\|T_{\vec{b}}(f)\right\|_{L^{1}} \\
& \leq C \lambda^{-1} \sum_{j=-\infty}^{\infty}\left|\lambda_{j}\right|
\end{aligned}
$$




$$
\leq C \lambda^{-1}\left(\sum_{j=-\infty}^{\infty}\left|\lambda_{j}\right|^{1 /(1+\beta)}\right)^{(1+\beta)} .
$$

Taking infinum over all the central atomic decomposition completes the proof of the theorem.

\section{Boundedness on HerZ TyPE HaRdy SPACES}

First, we introduce the definition of Herz type Hardy space and its atomic decomposition characteristic as follows.

Definition 6. Let $b_{i}(i=1, \cdots, m)$ be locally integrable functions, $1<q<\infty, \alpha \geq$ $1-1 / q$. A function $a$ is called a central $(\alpha, q, \vec{b})$-atom (or a central $(\alpha, q, \vec{b})$-atom of restricted type), if

(1) $\operatorname{supp} a \subset B=B\left(x_{0}, r\right)$ (or for some $r \geq 1$ ),

(2) $\|a\|_{L^{q}} \leq \mu(B)^{-\alpha}$,

(3) $\int_{B} a(x) d \mu(x)=\int_{B} a(x) \prod_{i \in \sigma} b_{i}(x) d \mu(x)=0$ for any $\sigma \in C_{i}^{m}, 1 \leq i \leq m$.

Definition 7. Let $\alpha \in R, 0<p<\infty$ and $1 \leq q<\infty$. For $k \in \mathbf{Z}$ and $x_{0} \in X$, set $B_{k}=\left\{x \in X: d\left(x_{0}, x\right) \leq 2^{k}\right\}$ and $C_{k}=B_{k} \backslash B_{k-1}$. Denote by $\chi_{k}$ the characteristic function of $C_{k}$ and by $\chi_{0}$ the characteristic function of $B_{0}$.

(1) The homogeneous Herz space is defined by

$$
\dot{K}_{q}^{\alpha, p}(X)=\left\{f \in L_{l o c}^{q}\left(X \backslash\left\{x_{0}\right\}\right):\|f\|_{\left.\dot{K}_{q}^{\alpha, p}<\infty\right\},},\right.
$$

where

$$
\|f\|_{\dot{K}_{q}^{\alpha, p}}=\left(\sum_{k=-\infty}^{\infty} \mu\left(B_{k}\right)^{\alpha p}\left\|f \chi_{k}\right\|_{L^{q}}^{p}\right)^{1 / p} .
$$

(2) The nonhomogeneous Herz space is defined by

$$
K_{q}^{\alpha, p}(X)=\left\{f \in L_{l o c}^{q}(X):\|f\|_{\left.K_{q}^{\alpha, p}<\infty\right\}},\right.
$$

where

$$
\|f\|_{K_{q}^{\alpha, p}}=\left[\sum_{k=1}^{\infty} \mu\left(B_{k}\right)^{\alpha p}\left\|f \chi_{k}\right\|_{L^{q}}^{p}+\left\|f \chi_{0}\right\|_{L^{q}}^{p}\right]^{1 / p} .
$$

A function $f$ is said to belong to the homogeneous $H \dot{K}_{q, \vec{b}}^{\alpha, p}(X)$ (or to the nonhomogeneous $H K_{q, \vec{b}}^{\alpha, p}(X)$ ), if it can be written as $f=\sum_{j=-\infty}^{\infty} \lambda_{j} a_{j}(x)$ (or $f=$ 
$\sum_{j=0}^{\infty} \lambda_{j} a_{j}(x)$ ), where $a_{j}$ is a central $(\alpha, q, \vec{b})$-atom (or a central $(\alpha, q, \vec{b})$-atom of restricted type) supported on $B\left(x_{0}, 2^{j}\right)$ and $\sum_{j=-\infty}^{\infty}\left|\lambda_{j}\right|^{p}<\infty\left(\right.$ or $\left.\sum_{j=0}^{\infty}\left|\lambda_{j}\right|^{p}<\infty\right)$. Moreover,

$$
\|f\|_{H \dot{K}_{q, \vec{b}}^{\alpha, p}}=\inf \left(\sum_{j=-\infty}^{\infty}\left|\lambda_{j}\right|^{p}\right)^{1 / p}\left(\operatorname{orinf}\left(\sum_{j=0}^{\infty}\left|\lambda_{j}\right|^{p}\right)^{1 / p}\right),
$$

where the infimum are taken over all of the above decompositions of $f$.

Definition 8 (see [11]). Let $\alpha \in \mathbf{R}, 0<p<\infty$ and $1 \leq q<\infty\left(B_{k}\right.$ and $C_{k}$ are the same as Definition 6).

(1) A measure function $f$ is said to belong to the homogeneous weak Herz space $W \dot{K}_{q}^{\alpha, p}(X)$, if

$$
\|f\|_{W \dot{K}_{q}^{\alpha, p}}=\sup _{\lambda>0} \lambda\left(\sum_{k=-\infty}^{+\infty} \mu\left(B_{k}\right)^{\alpha p} \mu\left(\left\{x \in C_{k}:|f(x)|>\lambda\right\}\right)^{p / q}\right)^{1 / p}<\infty ;
$$

(2) A measure function $f$ is said to belong to the inhomogeneous weak Herz space $W K_{q}^{\alpha, p}(X)$, if

$$
\begin{aligned}
\|f\|_{W K_{q}^{\alpha, p}=} & \sup _{\lambda>0} \lambda\left(\sum_{k=1}^{+\infty} \mu\left(B_{k}\right)^{\alpha p} \mu\left(\left\{x \in C_{k}:|f(x)|>\lambda\right\}\right)^{p / q}\right. \\
& \left.+\mu\left(\left\{x \in B_{0}:|f(x)|>\lambda\right\}\right)^{p / q}\right)^{1 / p}<\infty .
\end{aligned}
$$

Now we state our theorems.

Theorem 6. Let $b_{i} \in \operatorname{Lip}_{\beta_{i}}(X), 1 \leq i \leq m, \sum_{i=1}^{m} \beta_{i}=\beta, 0<\beta \leq 1,1 / q_{2}=$ $1 / q_{1}-\beta, 0<p<\infty$ and $1-1 / q_{1} \leq \alpha<1-1 / q_{1}+\delta / n_{0}$. Then the multilinear commutator $T_{\vec{b}}$ is bounded from $H \dot{K}_{q_{1}, \vec{b}}^{\alpha, p}(X)$ to $\dot{K}_{q_{2}}^{\alpha, p}(X)$.

Theorem 7. Let $b_{i} \in \operatorname{Lip}_{\beta_{i}}(X), 1 \leq i \leq m, \sum_{i=1}^{m} \beta_{i}=\beta, 0<\beta \leq \delta / n_{0}, 1 / q_{2}=$ $1 / q_{1}-\beta$ and let $0<p \leq 1$. Then the multilinear commutator $T_{\vec{b}}$ is bounded from $H \dot{K}_{q_{1}}^{\alpha, p}(X)$ to $W \dot{K}_{q_{2}}^{\alpha, p}(X)$.

Proof of Theorem 6. Let $f \in H \dot{K}_{q_{1}, \vec{b}}^{\alpha, p}(X)$ and let $f(x)=\sum_{j=-\infty}^{\infty} \lambda_{j} a_{j}(x)$ be the atomic decomposition of $f$ as in Definition 7 We write

$$
\begin{aligned}
& \left\|T_{\vec{b}}(f)(x)\right\|_{\dot{K}_{q_{2}}^{\alpha, p}}=\left(\sum_{k=-\infty}^{\infty} \mu\left(B_{k}\right)^{\alpha p}\left\|T_{\vec{b}}(f) \chi_{k}\right\|_{L^{q_{2}}}^{p}\right)^{1 / p} \\
& \leq C\left[\sum_{k=-\infty}^{\infty} \mu\left(B_{k}\right)^{\alpha p}\left(\sum_{j=-\infty}^{\infty}\left|\lambda_{j}\right|\left\|T_{\vec{b}}\left(a_{j}\right) \chi_{k}\right\|_{L^{q_{2}}}\right)^{p}\right]^{1 / p}
\end{aligned}
$$




$$
\begin{aligned}
& \leq C\left[\sum_{k=-\infty}^{\infty} \mu\left(B_{k}\right)^{\alpha p}\left(\sum_{j=-\infty}^{k-3}\left|\lambda_{j}\right||| T_{\vec{b}}\left(a_{j}\right) \chi_{k} \|_{L^{q_{2}}}\right)^{p}\right]^{1 / p} \\
& +C\left[\sum_{k=-\infty}^{\infty} \mu\left(B_{k}\right)^{\alpha p}\left(\sum_{j=k-2}^{\infty} \mid \lambda_{j}\left\|T_{\vec{b}}\left(a_{j}\right) \chi_{k}\right\|_{L^{q_{2}}}\right)^{p}\right]^{1 / p} \\
& =J+J J .
\end{aligned}
$$

For the term $J J$, by Hölder's inequality and as Theorem 1 states that $T_{\vec{b}}$ is bounded from $L^{q_{1}}(X)$ to $L^{q_{2}}(X)$, we have

$$
\begin{aligned}
& J J \leq C\left[\sum_{k=-\infty}^{\infty} \mu\left(B_{k}\right)^{\alpha p}\left(\sum_{j=k-2}^{\infty}\left|\lambda_{j}\right|\left\|T_{\vec{b}}\left(a_{j}\right) \chi_{k}\right\|_{L^{q_{2}}}\right)^{p}\right]^{1 / p} \\
& \leq C\|\vec{b}\|_{L i p_{\beta}} \\
& \times\left[\sum_{k=-\infty}^{\infty} \mu\left(B_{k}\right)^{\alpha p}\left(\sum_{j=k-2}^{\infty}\left|\lambda_{j}\right|\left\|a_{j} \chi_{k}\right\|_{L^{q_{1}}}\right)^{p}\right]^{1 / p} \\
& \leq C\|\vec{b}\|_{L i p_{\beta}}\left[\sum_{k=-\infty}^{\infty} \mu\left(B_{k}\right)^{\alpha p}\left(\sum_{j=k-2}^{\infty}\left|\lambda_{j}\right| \mu\left(B_{j}\right)^{-\alpha}\right)^{p}\right]^{1 / p} \\
& \leq C\|\vec{b}\|_{L i p_{\beta}} \\
& \times\left\{\begin{array}{c}
{\left[\sum_{j=-\infty}^{\infty}\left|\lambda_{j}\right|^{p} \sum_{k=-\infty}^{j+2}\left(\frac{\mu\left(B_{k}\right)}{\mu\left(B_{j}\right)}\right)^{\alpha p}\right]^{1 / p}, 0<p \leq 1} \\
{\left[\sum_{j=-\infty}^{\infty}\left|\lambda_{j}\right|^{p}\left(\sum_{k=-\infty}^{j+2}\left(\frac{\mu\left(B_{k}\right)}{\mu\left(B_{j}\right)}\right)^{\alpha p / 2}\right)\right.} \\
\left.\left(\sum_{k=-\infty}^{j+2}\left(\frac{\mu\left(B_{k}\right)}{\mu\left(B_{j}\right)}\right)^{\alpha p^{\prime} / 2}\right)^{p / p^{\prime}}\right]^{1 / p}, 1<p<\infty
\end{array}\right. \\
& \leq C\|\vec{b}\|_{L i p_{\beta}}\left\{\begin{array}{l}
{\left[\sum_{j=-\infty}^{\infty}\left|\lambda_{j}\right|^{p} \sum_{k=-\infty}^{j+2} A_{2}{ }^{(k-j) \alpha p}\right]^{1 / p}, 0<p \leq 1} \\
{\left[\sum_{j=-\infty}^{\infty}\left|\lambda_{j}\right|^{p}\left(\sum_{k=-\infty}^{j+2} A_{2}{ }^{(k-j) \alpha p / 2}\right)\right.} \\
\left.\left(\sum_{k=-\infty}^{j+2} A_{2}{ }^{(k-j) \alpha p^{\prime} / 2}\right)^{p / p^{\prime}}\right]^{1 / p}, 1<p<\infty
\end{array}\right.
\end{aligned}
$$




$$
\begin{aligned}
& \leq C\|\vec{b}\|_{L_{i p_{\beta}}}\left(\sum_{j=-\infty}^{\infty}\left|\lambda_{j}\right|^{p}\right)^{1 / p} \\
& \leq C\|f\|_{H \dot{K}_{q_{1}, \vec{b}}^{\alpha, p}}
\end{aligned}
$$

For the term $J$, let $C_{k}=B_{k} \backslash B_{k-1}, \chi_{k}=\chi_{C_{k}}, b_{j}^{i}=\mu\left(B_{j}\right)^{-1} \int_{B_{j}} b_{i}(x) d \mu(x), 1 \leq$ $i \leq m$. By Hölder's inequality, by the vanishing moment of atom $a$ and by the reverse doubling condition, we get, for $x \in 2^{k+1} B \backslash 2^{k} B$,

$$
\begin{aligned}
& \left|T_{\vec{b}}\left(a_{j}\right)(x)\right| \leq \int_{B_{j}} \prod_{i=1}^{m}\left|\left(b_{i}(x)-b_{i}(y)\right)\right|\left|K(x, y) \| a_{j}(y)\right| d \mu(y) \\
& \leq \int_{B_{j}} \prod_{i=1}^{m}\left(\mid b_{i}(x)-b_{i}\left(x_{0}\right)\right)|+|\left(b_{i}(y)-b_{i}\left(x_{0}\right) \mid\right)\left|K(x, y)-K\left(x, x_{0}\right)\right|\left|a_{j}(y)\right| d \mu(y) \\
& \leq C \prod_{i=1}^{m}\left|\left(b_{i}(x)-b_{i}\left(x_{0}\right)\right)\right| \int_{B_{j}} \frac{d\left(x_{0}, y\right)^{\delta}}{d\left(x, x_{0}\right)^{\delta} \mu\left(B\left(x_{0}, d\left(x, x_{0}\right)\right)\right)}\left|a_{j}(y)\right| d \mu(y) \\
& +\sum_{i=1}^{m-1} \sum_{\sigma \in C_{i}^{m}}\left|\left(\vec{b}(x)-\vec{b}\left(x_{0}\right)\right)_{\sigma}\right| \\
& \int_{B_{j}}\left|\left(\vec{b}(y)-\vec{b}\left(x_{0}\right)\right)_{\sigma_{c}}\right| \frac{d\left(x_{0}, y\right)^{\delta}}{d\left(x, x_{0}\right)^{\delta} \mu\left(B\left(x_{0}, d\left(x, x_{0}\right)\right)\right)}\left|a_{j}(y)\right| d \mu(y) \\
& +\prod_{i=1}^{m} \int_{B_{j}}\left|\left(b_{i}(y)-b_{i}\left(x_{0}\right)\right)\right| \frac{d\left(x_{0}, y\right)^{\delta}}{d\left(x, x_{0}\right)^{\delta} \mu\left(B\left(x_{0}, d\left(x, x_{0}\right)\right)\right)}\left|a_{j}(y)\right| d \mu(y) \\
& \leq C \frac{r_{j}^{\delta}}{d\left(x, x_{0}\right)^{\delta} \mu\left(B\left(x_{0}, d\left(x, x_{0}\right)\right)\right)} \cdot\left\{\|\vec{b}\|_{L i p_{\beta}} \rho\left(x, x_{0}\right)^{\beta}\left\|a_{j}\right\|_{L^{q_{1}}} \mu\left(B_{j}\right)^{1 / q_{1}^{\prime}}\right. \\
& +\sum_{i=1}^{m-1} \sum_{\sigma \in C_{i}^{m}}\left\|\vec{b}_{\sigma}\right\|_{L i p_{\beta}} \rho\left(x, x_{0}\right)^{\beta_{\sigma}} \int_{B_{j}}\left\|\vec{b}_{\sigma_{c}}\right\|_{L i p_{\beta}} \rho\left(y, x_{0}\right)^{\beta_{\sigma^{c}}}\left|a_{j}(y)\right| d \mu(y) \\
& \left.+\int_{B_{j}}\|\vec{b}\|_{L i p_{\beta}} \rho\left(y, x_{0}\right)^{\beta}\left|a_{j}(y)\right| d \mu(y)\right\} \\
& \leq C \frac{r_{j}^{\delta}}{d\left(x, x_{0}\right)^{\delta} \mu\left(B\left(x_{0}, d\left(x, x_{0}\right)\right)\right)}\|\vec{b}\|_{L i p_{\beta}} \rho\left(x, x_{0}\right)^{\beta}\left\|a_{j}\right\|_{L^{q_{1}}} \mu\left(B_{j}\right)^{1 / q_{1}^{\prime}} \\
& \leq C \frac{r_{j}^{\delta}}{d\left(x, x_{0}\right)^{\delta} \mu\left(B\left(x_{0}, d\left(x, x_{0}\right)\right)\right)}\|\vec{b}\|_{L i p_{\beta}} \rho\left(x, x_{0}\right)^{\beta} \mu\left(B_{j}\right)^{-\alpha+1 / q_{1}^{\prime}},
\end{aligned}
$$


SO

$$
\begin{gathered}
\left\|T_{\vec{b}}\left(a_{j}\right) \chi_{k}\right\|_{L^{q_{2}}} \\
\leq C\|\vec{b}\|_{L i p_{\beta}} \frac{r_{j}^{\delta}}{r_{k}^{\delta} \mu\left(B_{k}\right)} \mu\left(B_{j}\right)^{-\alpha+1 / q_{1}^{\prime}}\left(\int_{B_{k} \backslash B_{k-1}} \rho(x, u)^{\beta q_{2}} d \mu(x)\right)^{1 / q_{2}} \\
\leq C\|\vec{b}\|_{L i p_{\beta}} 2^{(j-k) \delta} \mu\left(B_{k}\right)^{-1 / q_{2}^{\prime}+\beta} \mu\left(B_{j}\right)^{-\alpha+1 / q_{1}^{\prime}}
\end{gathered}
$$

and

$$
\begin{aligned}
& J=C\left[\sum_{k=-\infty}^{\infty} \mu\left(B_{k}\right)^{\alpha p}\left(\sum_{j=-\infty}^{k-3}\left|\lambda_{j}\right||| T_{\vec{b}}\left(a_{j}\right) \chi_{k} \|_{L^{q_{2}}}\right)^{p}\right]^{1 / p} \\
& \leq C\|\vec{b}\|_{L i p_{\beta}}\left[\sum_{k=-\infty}^{\infty} \mu\left(B_{k}\right)^{\alpha p}\right. \\
& \left.\left(\sum_{j=-\infty}^{k-3}\left|\lambda_{j}\right| 2^{(j-k) \delta} \mu\left(B_{j}\right)^{1-1 / q_{1}-\alpha} \mu\left(B_{k}\right)^{-\left(1-1 / q_{2}-\beta\right)}\right)^{p}\right]^{1 / p} \\
& \leq C\|\vec{b}\|_{L i p_{\beta}}\left\{\begin{array}{c}
{\left[\sum_{j=-\infty}^{\infty}\left|\lambda_{j}\right|^{p} \sum_{k=j+3}^{\infty} 2^{(j-k) \delta p_{2}(j-k) n_{0}\left(1-1 / q_{2}-\alpha-\beta\right) p}\right]^{1 / p},} \\
\text { if } \quad 0<p \leq 1 \\
{\left[\sum_{j=-\infty}^{\infty}\left|\lambda_{j}\right|^{p}\left(\sum_{k=j+3}^{\infty} 2^{(j-k) \delta p / 2} 2^{(j-k) n_{0}\left(1-1 / q_{2}-\alpha-\beta\right) p / 2}\right)\right.} \\
\left.\times\left(\sum_{k=j+3}^{\infty} 2^{(j-k) \delta p^{\prime} / 2} 2^{(j-k) n_{0}\left(1-1 / q_{2}-\alpha-\beta\right) p^{\prime} / 2}\right)^{p / p^{\prime}}\right]^{1 / p} \\
\text { if } \quad 1<p<\infty
\end{array}\right. \\
& \leq C\|\vec{b}\|_{L i p_{\beta}}\left\{\begin{array}{c}
{\left[\sum_{j=-\infty}^{\infty}\left|\lambda_{j}\right|^{p} \sum_{k=j+3}^{\infty} 2^{(j-k) n_{0}\left(1-1 / q_{1}+\delta / n_{0}-\alpha\right) p}\right]^{1 / p},} \\
\text { if } 0<p \leq 1 \\
{\left[\sum_{j=-\infty}^{\infty}\left|\lambda_{j}\right|^{p}\left(\sum_{k=j+3}^{\infty} 2^{(j-k) n_{0}\left(1-1 / q_{1}+\delta / n_{0}-\alpha\right) p / 2}\right)\right.} \\
\left.\times\left(\sum_{k=j+3}^{\infty} 2^{(j-k) n_{0}\left(1-1 / q_{1}+\delta / n_{0}-\alpha\right) p^{\prime} / 2}\right)^{p / p^{\prime}}\right]^{1 / p}, \\
\text { if } 1<p<\infty
\end{array}\right. \\
& \leq C\|\vec{b}\|_{L i p_{\beta}}\left(\sum_{j=-\infty}^{\infty}\left|\lambda_{j}\right|^{p}\right)^{1 / p} \\
& \leq C\|f\|_{H \dot{K}_{q_{1}, \vec{b}}^{\alpha, p}} \text {. }
\end{aligned}
$$

This completes the proof of the theorem. 
Remark 1. Similar results related to nonhomogeneous weak Herz spaces also hold. The details are omitted in order to save space.

Proof of Theorem 7. We write $f=\sum_{j=-\infty}^{\infty} \lambda_{j} a_{j}$, where each $a_{j}$ is a central $\left(\alpha, q_{1}\right)$ atom supported on $B_{j}$ and $\sum_{j=-\infty}^{\infty}\left|\lambda_{j}\right|^{p}<\infty$. Write

$$
\begin{gathered}
\left\|T_{\vec{b}}(f)\right\|_{W \dot{K}_{q_{2}}^{\alpha, p}}^{p} \\
\leq \sup _{\lambda>0} \lambda^{p} \sum_{k=-\infty}^{\infty} \mu\left(B_{k}\right)^{\alpha p} \mu\left(\left\{x \in C_{k}:\left|T_{\vec{b}}\left(\sum_{j=k-1}^{\infty} \lambda_{j} a_{j}\right)(x)\right|>\lambda / 2\right\}\right)^{p / q_{2}} \\
+\sup _{\lambda>0} \lambda^{p} \sum_{k=-\infty}^{\infty} \mu\left(B_{k}\right)^{\alpha p} \mu\left(\left\{x \in C_{k}:\left|T_{\vec{b}}\left(\sum_{j=-\infty}^{k-2} \lambda_{j} a_{j}\right)(x)\right|>\lambda / 2\right\}\right)^{p / q_{2}} \\
=G_{1}+G_{2} .
\end{gathered}
$$

By the $\left(L^{q_{1}}, L^{q_{2}}\right)$ boundedness of $T_{\vec{b}}$ and by an estimate, similar to the one for $J J$ in Theorem 6, we get

$$
G_{1} \leq C \sum_{k=-\infty}^{\infty} \mu\left(B_{k}\right)^{\alpha p}\left\|T_{\vec{b}}\left(\sum_{j=k-1}^{\infty} \lambda_{j} a_{j}\right)(x) \chi_{k}\right\|_{L^{q_{2}}}^{p} \leq C\|\vec{b}\|_{\wedge_{\beta}}^{p} \sum_{j=-\infty}^{\infty}\left|\lambda_{j}\right|^{p} .
$$

For $G_{2}$, let us use the estimate

$$
\left|T_{\vec{b}}\left(a_{j}\right)(x)\right| \leq C\|\vec{b}\|_{L i p_{\beta}} \frac{r_{j}^{\delta}}{d\left(x, x_{0}\right)^{\delta} \mu\left(B\left(x_{0}, d\left(x, x_{0}\right)\right)\right)} \rho\left(x, x_{0}\right)^{\beta} \mu\left(B_{j}\right)^{-\alpha+1 / q_{1}^{\prime}},
$$

which was obtained in the estimate for $J$ in the proof of Theorem 6 . Notice that when $x \in C_{k}$,

$$
\begin{aligned}
\lambda<2 \sum_{j=-\infty}^{k-2}\left|\lambda_{j} \| T_{\vec{b}}\left(a_{j}\right)\right| & \leq C\|\vec{b}\|_{\text {Li }} \sum_{j=-\infty}^{k-2}\left|\lambda_{j}\right| \frac{r_{j}^{\delta}}{d\left(x, x_{0}\right)^{\delta} \mu\left(B\left(x_{0}, d\left(x, x_{0}\right)\right)\right)} \\
& \times \rho\left(x, x_{0}\right)^{\beta} \mu\left(B_{j}\right)^{-\alpha+1 / q_{1}^{\prime}} \\
& \leq C\|\vec{b}\|_{L i p_{\beta}} \sum_{j=-\infty}^{k-2}\left|\lambda_{j}\right| 2^{(j-k) \delta} \mu\left(B_{k}\right)^{-1+\beta} \mu\left(B_{j}\right)^{-\alpha+1 / q_{1}^{\prime}} \\
& \leq C\|\vec{b}\|_{L i p_{\beta}} \sum_{j=-\infty}^{k-2}\left|\lambda_{j}\right| 2^{(j-k) \delta} \mu\left(B_{k}\right)^{-\alpha-1 / q_{2}} .
\end{aligned}
$$

For $\lambda>0$, choosing $k_{0} \in Z$ such that $B_{k_{0}}$ is the maximal set satisfying

$$
\mu\left(B_{k_{0}}\right)^{\alpha+1 / q_{2}} \leq C\|\vec{b}\|_{L i p_{\beta}} \lambda^{-1}\left(\sum_{j=-\infty}^{\infty}\left|\lambda_{j}\right|^{p}\right)^{1 / p}
$$


then if $k>k_{0}$, it is easy to see that

$$
\left\{x \in C_{k}:\left|T_{\vec{b}}\left(\sum_{j=-\infty}^{k-2} \lambda_{j} a_{j}\right)(x)\right|>\lambda / 2\right\}
$$

is an empty set, thus

$$
\begin{aligned}
G_{2} & \leq \sup _{\lambda>0} \lambda^{p} \sum_{k=-\infty}^{k_{0}} \mu\left(B_{k}\right)^{\left(\alpha+1 / q_{2}\right) p} \\
& \leq C\|\vec{b}\|_{L i p_{\beta}}^{p} \sum_{j=-\infty}^{k-2}\left|\lambda_{j}\right|^{p} 2^{(j-k) \delta p} \mu\left(B_{k}\right)^{-\left(\alpha+1 / q_{2}\right) p} \sum_{k=-\infty}^{k_{0}} \mu\left(B_{k}\right)^{\left(\alpha+1 / q_{2}\right) p} \\
& \leq C\|\vec{b}\|_{L i p_{\beta}}^{p} \sum_{k=-\infty}^{k_{0}} \sum_{j=-\infty}^{k-2}\left|\lambda_{j}\right|^{p} 2^{(j-k) \delta p} \\
& \leq C\|\vec{b}\|_{L i p_{\beta}}^{p} \sum_{j=-\infty}^{\infty}\left|\lambda_{j}\right|^{p} .
\end{aligned}
$$

So, we get,

$$
\left\|T_{\vec{b}}(f)\right\|_{W \dot{K}_{q_{2}}^{\alpha, p}} \leq C\|\vec{b}\|_{L i p_{\beta}}\left(\sum_{j=-\infty}^{\infty}\left|\lambda_{j}\right|^{p}\right)^{1 / p} .
$$

Taking the infinum, we have completed the proof.

\section{REFERENCES}

[1] J. Alvarez, "Continuity properties for linear commutators of calderón-zygmund operators," Collect. Math., vol. 1998, no. 1, pp. 17-31, 49.

[2] J. Alvarez, R. J. Bagby, D. S. Kurtz, and C. Pérez, "Weighted estimates for commutators of linear operators," Studia Math., vol. 104, no. 2, pp. 195-209, 1993.

[3] A. Bernardis, S. Hartzstein, and G. Pradolini, "Weighted inequalities for commutators of fractional integrals on spaces of homogeneous type," J. Math. Anal. Appl., vol. 322, no. 2, pp. 825-846, 2006.

[4] J. J. Betancor, "A commutator theorem for fractional integrals in spaces of homogeneous type," Int. J. Math. Math. Sci., vol. 24, no. 6, pp. 403-418, 2000.

[5] M. Bramanti and M. C. Cerutti, "Commutators of singular integrals on homogeneous spaces," Boll. Un. Mat. Ital. B (7), vol. 10, no. 4, pp. 843-883, 1996.

[6] S. Chanillo, "A note on commutators," Indiana Univ. Math. J., vol. 31, no. 1, pp. 7-16, 1982.

[7] Q. Chen and L. Liu, "Continuity for multilinear integral operators on some hardy and herz type spaces," Int. J. Open Probl. Comput. Sci. Math., vol. 2, no. 2, pp. 215-230, 2009.

[8] W. Chen and E. Sawyer, "Endpoint estimates for commutators of singular integrals on spaces of homogeneous type," J. Math. Anal. Appl., vol. 282, no. 2, pp. 553-566, 2003.

[9] R. R. Coifman, R. Rochberg, and G. Weiss, "Factorization theorems for hardy spaces in several variables," Ann. of Math. (2), vol. 103, no. 3, pp. 611-635, 1976. 
[10] A. E. Gatto and S. Vági, Fractional integrals on spaces of homogeneous type, ser. Lecture Notes in Pure and Appl. Math. New York: Marcel Dekker, Inc., 1990, vol. 122.

[11] I. Genebashvili, A. Gogatishvili, V. Kokilashvili, and M. Krbec, Weight theory for integral transforms on spaces of homogeneous type, ser. Pitman Monographs and Surveys in Pure and Applied Mathematics. Harlow: Longman, 1998, vol. 92.

[12] Y. Han and D. Yang, "New characterizations and applications of inhomogeneous besov and triebellizorkin spaces on homogeneous type spaces and fractals," Dissertationes Math. (Rozprawy Mat.), vol. 403, p. 102, 2002.

[13] R. A. Macías and C. Segovia, "Lipschitz functions on spaces of homogeneous type," Adv. in Math., vol. 33, no. 3, pp. 257-270, 1979.

[14] G. Pradolini and O. Salinas, "Commutators of singular integrals on spaces of homogeneous type," Czechoslovak Math. J., vol. 57(132), no. 1, pp. 75-93, 2007.

[15] E. M. Stein, Harmonic analysis: real-variable methods, orthogonality, and oscillatory integrals, ser. Princeton Mathematical Series. Princeton: Princeton University Press, 1993, vol. 43.

[16] M. Wang and L. Liu, "Lipschitz estimates for multilinear commutator of littlewood-paley operator," Acta Math. Acad. Paedagog. Nyházi. (N. S.), vol. 25, no. 1, pp. 85-104, 2009.

\section{Author's address}

Chen Yue and Liu Lanzhe

Department of Mathematics, Changsha University of Science and Technolegy, Changsha 410077, P.R.of China

E-mail address: lanzheliu@163.com 